\title{
Flexible Multivariate GARCH Modeling With an Application to International Stock Markets
}

\author{
Olivier Ledoit \\ Equities Division \\ Credit Suisse First Boston \\ Pedro Santa-Clara * \\ The Anderson School \\ UCLA \\ Michael Wolf ${ }^{\dagger}$ \\ Department of Economics \\ Universitat Pompeu Fabra
}

April 2002

*Phone: 310-206-6077, fax: 310-206-5455, e-mail: pedro.santa-clara@anderson.ucla.edu.

${ }^{\dagger}$ Research supported by DGES grant BEC2001-1270. This paper was finished while the third author was visiting Bocconi University whose hospitality is gratefully acknowledged.

We thank Frank de Jong, Bruno Gérard, Hans Mikkelsen, and Robert Whitelaw for comments. We are grateful to the editor John Campbell and two anonymous referees for suggestions and constructive criticisms that have led to an improvement of the paper. Ilya Sharapov was extremely helpful with the numerical computations. All errors are our own. 


\title{
Flexible Multivariate GARCH Modeling With an Application to International Stock Markets
}

\begin{abstract}
The goal of this paper is to estimate time-varying covariance matrices. Since the covariance matrix of financial returns is known to change through time and is an essential ingredient in risk management, portfolio selection, and tests of asset pricing models, this is a very important problem in practice. Our model of choice is the Diagonal-Vech version of the Multivariate $\operatorname{GARCH}(1,1)$ model. This is the most straightforward multivariate extension of the $\operatorname{GARCH}(1,1)$ model, which is the standard model in univariate volatility estimation. Unfortunately, the estimation of the general Diagonal-Vech model model has proved to be numerically infeasible for dimensions higher than 5 . The common approach has been to estimate more restrictive models which are tractable but may not conform to the data. Our contribution is to propose an alternative estimation method that is numerically feasible for large-scale problems, produces positive semi-definite conditional covariance matrices, and does not impose unrealistic a priori restrictions. We provide an empirical application in the context of international stock markets, comparing the new estimator to a number of existing ones.
\end{abstract}

JEL CLASSIFICATION NOS: C13, C51, C61, G11, G15 


\section{Introduction}

The goal of this paper is to estimate conditional covariance matrices. Since the covariance matrix is an essential ingredient in risk management, portfolio selection, and tests of asset pricing models, this is a very important problem in practice. Estimating conditional covariance matrices is a multivariate extension of the simpler problem of estimating conditional variances. In the univariate case, many methods are available, ranging from the simple 'rolling window' estimation method to the sophisticated models of latent stochastic volatility. The most popular method, however, to estimate conditional variances is the GARCH(1,1) model. We do not claim that it is the "best" method, because a method that is uniformly better than the others does not seem to exist. On the other hand, many studies have shown that the univariate $\operatorname{GARCH}(1,1)$ gives reasonable results and it can be safely assumed that it will remain in use for some time to come; for example, see Andersen, Bollerslev, and Lange (1999) and Lee and Saltoğlu (2001). For these reasons, multivariate extensions of the univariate $\operatorname{GARCH}(1,1)$ model have long been of interest.

The most general multivariate model commonly considered is defined by:

$$
\begin{aligned}
\mathrm{E}\left[x_{i, t} \mid \Omega_{t-1}\right] & =0 \\
\operatorname{Cov}\left[x_{i, t}, x_{j, t} \mid \Omega_{t-1}\right] & =h_{i j, t}=c_{i j}+a_{i j} x_{i, t-1} x_{j, t-1}+b_{i j} h_{i j, t-1}
\end{aligned}
$$

where $\Omega_{t-1}$ denotes the conditioning information set available at time $t-1$ and $x_{i, t}$ denotes the realization of the $i$ th variable $(i=1, \ldots, N)$ at time $t$. The parameter values satisfy

$a_{i j}, b_{i j} \geq 0 \forall i, j=1, \ldots, N$ and $c_{i i}>0 \forall i=1, \ldots, N$. Equation (2) is known as the Diagonal-Vech model. It assumes that the conditional covariance of variables $x_{i}$ and $x_{j}$ depends on its lagged value and on past realizations of the product $x_{i} x_{j}$ only (Bollerslev, Engle and Wooldridge, 1988). Also, Equation (1) assumes that the variables have zero conditional mean, which can always be justified by taking them to be residuals coming from some regression model. While more general models can be thought of, they involve too many parameters to be of practical interest.

The natural way to estimate the conditional covariance matrix is to compute the (quasi) maximum likelihood estimates of the parameters $c_{i j}, a_{i j}$, and $b_{i j}$ from observations of all the variables in the vector $x$. Unfortunately, this is not computationally feasible for matrices of dimension $N>5$ (Ding and Engle, 1994): there are too many parameters, $3 N(N+1) / 2$, and they interact in a way that is too intricate for existing optimization algorithms to converge. Another problem is that the estimation of the general Diagonal-Vech model does not necessarily yield conditional covariance matrices that are positive semi-definite.

The existing literature avoids these difficulties by imposing additional structure on the problem. For example, Ding and Engle (1994) give a sequence of 20 nested models that are particular cases of (2), by specifying, for example, that the conditional correlations should be constant, or that there is some factor structure in the conditional covariance matrix. Additional models can be found in Engle and Kroner (1995), Engle and Mezrich (1996), and Engle (2000), among others. Apart from being tractable, these models typically also 
ensure that the resulting conditional covariance matrices are positive semi-definite. Imposing additional structure is fine if we have a priori reasons to believe in it, but that is rarely the case. In order to avoid specification error, it is typically preferable to "let the data speak", which means finding a way to estimate the unrestricted model.

Our basic idea proceeds in two steps. The first step is to obtain each set of coefficient estimates $\hat{c}_{i j}, \hat{a}_{i j}$, and $\hat{b}_{i j}$ separately for every $(i, j)$. This can be achieved simply by estimating a two-dimensional or one-dimensional $\operatorname{GARCH}(1,1)$ model (for $i \neq j$ or $i=j$ respectively), which is computationally feasible using a traditional method such as maximum likelihood. We bring together the outputs of these separate estimation procedures into matrices $\hat{C}=\left[\hat{c}_{i j}\right]_{i, j=1, \ldots, N}, \hat{A}=\left[\hat{a}_{i j}\right]_{i, j=1, \ldots, N}$, and $\hat{B}=\left[\hat{b}_{i j}\right]_{i, j=1, \ldots, N}$. However, the coefficient matrices $\hat{C}, \hat{A}$, and $\hat{B}$ are generally incompatible with each other in the sense that they yield conditional covariance matrices that are not positive semi-definite. Therefore, our second step is to transform the estimated parameter matrices $\hat{C}, \hat{A}$, and $\hat{B}$ in such a way that they yield conditional covariance matrices that are guaranteed to be positive semi-definite, where the transformation is chosen to be the least disruptive possible (according to some metric). In addition, we obtain $\operatorname{GARCH}(1,1)$ parameters that correspond to covariance-stationary processes, in contrast to the implicit model behind the exponential smoothing scheme that is quite popular for large-dimensional covariance matrices and used by RiskMetrics, for example.

In summary, the main advantage of our estimation method is that it is the first to allow estimation of the full-blown Diagonal-Vech model for dimensions larger than $N=5$ without imposing any a priori restrictions. Our conditional covariance matrices are only forced to be positive semi-definite, but they generally turn out to be positive definite and wellconditioned, which is a characteristic that (purely on economic grounds) we would expect from the true covariance matrix (as long as we consider a menu of non-redundant assets). An additional advantage is the reduced computational cost compared to traditional multivariate models; see Subsection 3.3.

The paper proceeds as follows. Section 2 develops the new estimation method. Section 3 gives an empirical application to international stock markets. Section 4 concludes. An appendix highlights some computational issues. All tables and figures appear at the end of the paper.

\section{Estimation Method}

It is important to understand precisely why it is so difficult to estimate the unrestricted Diagonal-Vech model in Equation (2) by maximum likelihood. Although there are many parameters, $3 N(N+1) / 2$, this cannot be the only source of the problem. The number of parameters in the unconditional covariance matrix is of the same order of magnitude, $N(N+1) / 2$, and estimating the unconditional covariance matrix by the sample covariance matrix (which is the maximum likelihood estimator under normality) is computationally trivial. Computing the sample covariance matrix is easy because it can be done in a 
"decentralized" fashion: for every variable, compute its sample variance (this is a univariate problem) and insert it into the diagonal; for every pair of variables compute their sample covariance (this is a bivariate problem) and insert it at the appropriate place off the diagonal. Thus, a large sample covariance matrix can be constructed by solving $N(N+1) / 2$ univariate or bivariate estimation problems. This is what keeps computations feasible (as opposed to solving a single, large multivariate estimation problem).

Could the same "decentralized" process be used to compute the Diagonal-Vech estimator? Not directly. The crucial problem is the compatibility of the parameters that come out of all the univariate or bivariate estimations. To pursue our analogy, in the case of the sample covariance matrix, the compatibility constraint is that the matrix be positive semidefinite. It turns out that there exists a mathematical result that guarantees that the sample covariance matrix constructed by putting together the individual sample variances and sample covariances is positive semi-definite. This is very fortunate, and comes out of the simplicity of the setting. For the Diagonal-Vech model, positive definiteness is not automatic.

The rest of this section develops an approach to deal with this problem.

\subsection{Decentralized Estimation of Multivariate $\operatorname{GARCH}(1,1)$}

Consider what happens when we try to decentralize the estimation process for the DiagonalVech model. As we said, this constitutes the first step of our estimation procedure. This step itself can be divided into two sub-steps, corresponding to the estimation of the diagonal and the off-diagonal coefficients, respectively.

\subsubsection{Diagonal Coefficients}

We estimate a univariate $\operatorname{GARCH}(1,1)$ process for every one of the variables by maximum likelihood and we get consistent estimators $\hat{c}_{i i}, \hat{a}_{i i}$, and $\hat{b}_{i i}$. Separately for each $i=1, \ldots, N$, we solve the quasi-likelihood maximization program, assuming conditional normality:

$$
\max _{c_{i i}, a_{i i}, b_{i i}} \prod_{t=1}^{T} \frac{1}{\sqrt{2 \pi h_{i i, t}}} e^{-x_{i t}^{2} /\left(2 h_{i i, t}\right)} \quad \text { s.t. } \quad h_{i i, t}=c_{i i}+a_{i i} x_{i, t-1}^{2}+b_{i i} h_{i i, t-1} .
$$

For each $i$, we have a simple univariate $\operatorname{GARCH}(1,1)$ estimation problem, which many commercial packages solve quickly. The estimator is in general not efficient, as the conditional distribution may be different from normal, but it is consistent (e.g., Campbell, Lo, and MacKinlay, 1997, Section 12.2).

\subsubsection{Off-Diagonal Coefficients}

From above, we get parameter estimates $\hat{c}_{i i}, \hat{a}_{i i}$, and $\hat{b}_{i i}$. We can use them to construct conditional variance estimates $\hat{h}_{i i t}$. In the second stage, we use these estimates to specify 
quasi-likelihood functions for the off-diagonal elements. Separately for each $i=1, \ldots, N$ and $j=i+1, \ldots, N$, we solve:

$$
\begin{gathered}
\max _{c_{i j}, a_{i j}, b_{i j}} \prod_{t=1}^{T} \frac{1}{2 \pi \sqrt{\operatorname{det}\left(H_{i j, t}\right)}} e^{-X_{i j, t}^{\prime} H_{i j, t}^{-1} X_{i j, t} / 2} \\
\text { s.t. } X_{i j, t}=\left[\begin{array}{l}
x_{i, t} \\
x_{j, t}
\end{array}\right], H_{i j, t}=\left[\begin{array}{ll}
\hat{h}_{i i, t} & h_{i j, t} \\
h_{i j, t} & \hat{h}_{j j, t}
\end{array}\right] \text { and } h_{i j, t}=c_{i j}+a_{i j} x_{i, t-1} x_{j, t-1}+b_{i j} h_{i j, t-1} \text {. }
\end{gathered}
$$

This quasi-likelihood is obtained by restricting attention to the $2 \times 2$ submatrix of variables $x_{i}$ and $x_{j}$, fixing the conditional variances at their first-stage values $\hat{h}_{i i}$ and $\hat{h}_{j j}$, and assuming normality. As on the diagonal, quasi-likelihood theorems ensure consistency. Problem (3)-(4) is easy to solve using standard optimization algorithms, since there are only three free parameters. The positive definiteness of the conditional covariance submatrix $H_{i j, t}$ is ensured by imposing the following bounds in the estimation process: $\left|c_{i j}\right| \leq\left(\hat{c}_{i i} \hat{c}_{j j}\right)^{1 / 2}$, $0 \leq a_{i j} \leq\left(\hat{a}_{i i} \hat{a}_{j j}\right)^{1 / 2}$, and $0 \leq b_{i j} \leq\left(\hat{b}_{i i} \hat{b}_{j j}\right)^{1 / 2}$, as Ding and Engle (1994) show.

\subsection{Compatibility Constraints}

As noted before, the estimators of the coefficients $c_{i j}, a_{i j}$, and $b_{i j}$ obtained separately for every $(i, j)$ in Subsection 2.1 are not compatible with one another in the sense that the forecasted covariance matrix may not be positive definite. This subsection analyzes the mathematical relations that they must satisfy in order to become compatible.

\subsubsection{Positive Semi-Definite Conditional Covariance Matrix}

Following the notation of Ding and Engle (1994), let $C=\left[c_{i j}\right]_{i, j=1, \ldots, N}, A=\left[a_{i j}\right]_{i, j=1, \ldots, N}$, and $B=\left[b_{i j}\right]_{i, j=1, \ldots, N}$ denote matrices containing the parameters of the model. Let $H_{t}=\left[h_{i j t}\right]_{i, j=1, \ldots, N}$ denote the conditional covariance matrix at time $t$. Denote the matrix of cross-products of variables observed at time $t$ by: $\Sigma_{t}=\left[x_{i, t} x_{j, t}\right]_{i, j=1, \ldots, N}$. Then Equation (2) can be rewritten as:

$$
H_{t}=C+A * \Sigma_{t-1}+B * H_{t-1}
$$

where the symbol $*$ denotes the Hadamard product of two matrices. The Hadamard product of two matrices $U=\left[u_{i j}\right]_{i, j=1, \ldots, N}$ and $V=\left[v_{i j}\right]_{i, j=1, \ldots, N}$ is defined as the elementwise product $U * V=\left[u_{i j} v_{i j}\right]_{i, j=1, \ldots, N}$. Similarly, let $\div$ denote the elementwise division: $U \div V=$ $\left[u_{i j} / v_{i j}\right]_{i, j=1, \ldots, N}$ and let $\wedge$ denote the elementwise exponentiation: $U^{\wedge p}=\left[u_{i j}^{p}\right]_{i, j=1, \ldots, N}$.

Ding and Engle (1994) show that a sufficient condition to guarantee that the conditional covariance matrix $H_{t}$ is positive semi-definite almost surely (a.s.) is that $C, A$ and $B$ be positive semi-definite. We derive a somewhat weaker sufficient condition.

Proposition 1 If $C \div(1-B), A$ and $B$ are positive semi-definite, then the conditional covariance matrix is positive semi-definite. 
Proof of Proposition 1 Replacing recursively Equation (5) into itself yields:

$$
\begin{aligned}
H_{t} & =\sum_{k=0}^{\infty} B^{\wedge k} * C+\sum_{k=0}^{\infty} B^{\wedge k} * A * \Sigma_{t-k-1} \\
& =C \div(1-B)+\sum_{k=0}^{\infty} B^{\wedge k} * A * \Sigma_{t-k-1}
\end{aligned}
$$

The Hadamard product of two positive semi-definite matrices is positive semi-definite; for example, see Styan (1973). In addition, the sum of two positive semi-definite matrices is positive semi-definite. Finally, the matrix of cross products of realizations $\Sigma_{t-k-1}$ is positive semi-definite a.s. by construction. Therefore, the inspection of equation (6) shows that, under the conditions stated in Proposition 1, the conditional covariance matrix $H_{t}$ is guaranteed to be positive semi-definite a.s.

A simple example for which our condition holds but not the one in Ding and Engle (1994) is given by:

$$
B=\left[\begin{array}{cc}
0.9 & 0.84 \\
0.84 & 0.8
\end{array}\right] \text { and } C=\left[\begin{array}{cc}
1.0 & 1.1 \\
1.1 & 1.0
\end{array}\right]
$$

It is easy to check that here $C \div(1-B)$ is positive semi-definite but $C$ is not. While this example may or may not be economically relevant, it illustrates that the sufficient condition of Ding and Engle (1994) can indeed be weakened.

\section{Proposition 2 If:}

- the conditional multivariate distribution of the vector $x_{t}$ is continuous with unbounded support for all $t$;

- $\forall i, \forall j, b_{i j}<1$;

- the conditional covariance matrix $H_{t}$ is positive semi-definite a.s. for all $t$,

then it is necessary that the parameter matrix $C \div(1-B)$ be positive semi-definite.

Proof of Proposition 2 We make a proof by contradiction. Suppose that $C \div(1-B)$ has at least one negative eigenvalue $\lambda<0$. Expand the conditional covariance matrix as:

$$
H_{t}=C \div(1-B)+\sum_{k=0}^{t-1} B^{\wedge k} * A * \Sigma_{t-k-1}+B^{\wedge t} *\left[H_{0}-C \div(1-B)\right]
$$

Let maxeig $(\cdot)$ denote the maximum eigenvalue of a matrix. Since all the elements of $B$ have absolute value strictly below one, we have:

$$
\begin{aligned}
B^{\wedge t} & \rightarrow 0 \text { as } t \rightarrow \infty \\
B^{\wedge t} *\left[H_{0}-C \div(1-B)\right] & \rightarrow 0 \text { as } t \rightarrow \infty \\
\operatorname{maxeig}\left(B^{\wedge t} *\left[H_{0}-C \div(1-B)\right]\right) & \rightarrow 0 \text { as } t \rightarrow \infty
\end{aligned}
$$


Therefore, there exists a $T$ large enough such that maxeig $\left(B^{\wedge T} *\left[H_{0}-C \div(1-B)\right]\right)<-\lambda / 2$. The matrix $A *\left(x x^{\prime}\right)$ goes to the null matrix as the vector $x$ goes to the null vector. Hence, there exists a neighborhood $N_{0}$ of the null vector such that $\forall x \in N_{0}$, $\operatorname{maxeig}\left(A *\left(x x^{\prime}\right)\right)<-\lambda /(2 T)$. Since all the elements of $B$ have absolute value strictly below one, it implies: $\forall x \in N_{0}, \forall k=0, \ldots, T-1$, maxeig $\left(B^{\wedge k} * A *\left(x x^{\prime}\right)\right)<-\lambda /(2 T)$. In the event that $\forall k=0, \ldots, T-1, x_{k} \in N_{0}$, we have:

$$
\operatorname{maxeig}\left\{\sum_{k=0}^{T-1} B^{\wedge k} * A * \Sigma_{t-k-1}+B^{\wedge T} *\left[H_{0}-C \div(1-B)\right]\right\}<-\lambda .
$$

Therefore, by equation (7) the conditional covariance matrix $H_{T}$ is not positive semidefinite if this event occurs. Since the conditional multivariate distribution of the vector $x_{t}$ is continuous with unbounded support for all $t$, the event has a positive probability of happening, which leads to a contradiction. This proves that $C \div(1-B)$ cannot have any strictly negative eigenvalue.

The assumption that the elements of $B$ have absolute value strictly below one is innocuous since it comes from the variance and covariance stationarity of the Multivariate $\mathrm{GARCH}(1,1)$ process. Similarly, we can prove that the positive semi-definiteness of the parameter matrix $A$ is also a necessary condition.

Proposition 3 If the conditional multivariate distribution of the vector $x_{t}$ is continuous with unbounded support and $H_{t}$ is positive semi-definite a.s. for all t, then it is necessary that the parameter matrix $A$ be positive semi-definite.

Proof of Proposition 3 Again we make the proof by contradiction. Let mineig(.) denote the smallest eigenvalue of a matrix. Suppose that $A$ has at least one negative eigenvalue, that is, mineig $(A)=\lambda<0$. Recall the Multivariate $\operatorname{GARCH}(1,1)$ recursion $H_{t}=$ $C+A *\left(x_{t-1} x_{t-1}^{\prime}\right)+B * H_{t-1}$. We work conditionally on $H_{t-1}$. If $x_{t-1}$ is equal to the unit vector then mineig $\left[A *\left(x_{t-1} x_{t-1}^{\prime}\right)\right]=\lambda$. Therefore, by continuity, there exists a neighborhood $N_{1}$ of the unit vector such that $\forall x_{t-1} \in N_{1}$, mineig $\left[A *\left(x_{t-1} x_{t-1}^{\prime}\right)\right]<\lambda / 2$. Let $\mu=\operatorname{maxeig}\left(C+B * H_{t-1}\right)$. In the event that $x_{t-1}=\sqrt{-2 \mu / \lambda} z$ for some $z \in N_{1}$, we have: mineig $\left[C+A *\left(x_{t-1} x_{t-1}^{\prime}\right)+B * H_{t-1}\right]<0$. Therefore, by the recursion formula the conditional covariance matrix $H_{t}$ is not positive semi-definite if this event occurs. Since the conditional multivariate distribution of the vector $x_{t-1}$ is continuous with unbounded support, the event has a positive probability of happening, which leads to a contradiction. This proves that $A$ cannot have any strictly negative eigenvalue.

For $B$, the situation is less clear. Technically speaking, the only necessary condition is that $B^{\wedge k} * A$ be positive semi-definite for all $k \geq 1$. It is possible to construct a counter-example with a matrix $B$ that satisfies this necessary condition but is not positive semi-definite. In the univariate case, just take: $A=0, B=-1$. This counter-example is mathematically correct but economically degenerate, and we have not been able to construct a more realistic 
one. In general, after extensive numerical experiments, our overall feeling is that the couples $(A, B)$ that satisfy the necessary condition and where $B$ is not positive semi-definite are extremely rare, and can perhaps be ruled out on economic grounds. However, we have not been able to prove any formal result along these lines. Hence, we will make the positive definiteness of $B$ an assumption rather than a conclusion.

Assumption 1 The true coefficient matrix $B$ in the Multivariate GARCH(1,1) model is positive semi-definite.

\subsubsection{Covariance Stationarity}

Another common concern in the application of GARCH models to financial returns is that the fitted model be covariance stationary. Hence, we want to make sure that $a_{i j}+b_{i j}<$ $1, \forall i, j=1, \ldots, N$. The following proposition shows that it is only necessary to verify this on the diagonal, as long as the coefficient matrices are positive semi-definite.

Proposition 4 If $A$ and $B$ are positive semi-definite and if

$$
a_{i i}+b_{i i}<1 \quad \forall i=1, \ldots, N
$$

then

$$
a_{i j}+b_{i j}<1 \quad \forall i, j=1, \ldots, N
$$

\section{Proof of Proposition 4}

$$
a_{i j}+b_{i j}=\left|a_{i j}\right|+\left|b_{i j}\right| \leq \sqrt{a_{i i} a_{j j}}+\sqrt{b_{i i} b_{j j}} \leq \sqrt{a_{i i}+b_{i i}} \sqrt{a_{j j}+b_{j j}}
$$

where the second to last inequality is a consequence of $A$ and $B$ being positive semi-definite and the last inequality is a consequence of the Hölder inequality.

In conclusion, the definitive version of our set of compatibility constraints is: $C \div(1-B)$, $A$ and $B$ positive semi-definite, and $a_{i}+b_{i}<1, \forall i=1, \ldots, N$.

\subsection{Transformation of Coefficient Matrices}

We now build the matrices $\hat{C}=\left[\hat{c}_{i j}\right]_{i, j=1, \ldots, N}, \hat{A}=\left[\hat{a}_{i j}\right]_{i, j=1, \ldots, N}$, and $\hat{B}=\left[\hat{b}_{i j}\right]_{i, j=1, \ldots, N}$ by placing the estimators from Section 2.1.1 on the diagonal and placing the estimators from Section 2.1.2 on the appropriate positions off the diagonal. For convenience, we also define $D=C \div(1-B)$ and $\hat{D}=\hat{C} \div(1-\hat{B})$. Note that $\hat{D}$ thus defined $i s$ the quasi-maximum likelihood estimator of $D$.

$\hat{D}, \hat{A}$ and $\hat{B}$ are consistent estimators of $D, A$ and $B$ respectively, but they are generally not positive semi-definite. To be precise, $\hat{D}, \hat{A}$ and $\hat{B}$ converge to positive semi-definite matrices 
(under Assumption 1), but in finite sample there is no guarantee that they are positive semidefinite. Practically speaking, our experience has been that, for reasonable sample sizes, finding positive semi-definite estimates is extremely rare. In other words, this decentralized procedure yields parameters that are not compatible with one another. This is why it has not been used in the existing literature, and why further restrictions are commonly imposed on the Diagonal-Vech model.

Our central innovation is to transform the estimators $\hat{D}, \hat{A}$ and $\hat{B}$ to positive semi-definite matrices $\tilde{D}, \tilde{A}$ and $\tilde{B}$, which we then take to be the estimates of $D, A$, and $B$. These matrices $\tilde{D}, \tilde{A}$ and $\tilde{B}$ are chosen to be the closest to $\hat{D}, \hat{A}$ and $\hat{B}$, respectively, according to a certain norm, but forcing the diagonal parameters obtained from univariate $\operatorname{GARCH}(1,1)$ estimation to remain unchanged. This can be formalized as:

$$
\begin{aligned}
& \min _{\tilde{D}}\|\tilde{D}-\hat{D}\| \quad \text { s.t. } \quad \tilde{D} \text { is positive semi-definite and } \tilde{d}_{i i}=\hat{d}_{i i}, \forall i=1, \ldots, N \\
& \min _{\tilde{A}}\|\tilde{A}-\hat{A}\| \quad \text { s.t. } \quad \tilde{A} \text { is positive semi-definite and } \tilde{a}_{i i}=\hat{a}_{i i}, \forall i=1, \ldots, N \\
& \min _{\tilde{B}}\|\tilde{B}-\hat{B}\| \quad \text { s.t. } \quad \tilde{B} \text { is positive semi-definite and } \tilde{b}_{i i}=\hat{b}_{i i}, \forall i=1, \ldots, N
\end{aligned}
$$

Once we have $\tilde{D}$ and $\tilde{B}$, we can recalculate $\tilde{C}=\tilde{D} *(1-\tilde{B})$.

One appealing property of this transformation is that it guarantees that the multivariate $\operatorname{GARCH}(1,1)$ process will not explode, that is, $\left|\tilde{a}_{i j}+\tilde{b}_{i j}\right|<1, \forall i, j=1, \ldots, N$. As shown in Proposition 4, it is sufficient to check the diagonal, since the transformed matrices are by construction positive semi-definite. Since we preserve the diagonal elements of $\hat{A}$ and $\hat{B}$, which come from covariance-stationary univariate $\operatorname{GARCH}(1,1)$ processes, this condition is automatically verified.

Another useful property is that the conditional covariance matrix $H_{t}$ is in general invertible. The parameter matrices $\tilde{D}, \tilde{A}$ and $\tilde{B}$ are not invertible because, by construction, they lie on the frontier of the convex set of positive semi-definite matrices, and only the interior of this set is made of invertible matrices. Nonetheless, combining $\tilde{D}, \tilde{A}$ and $\tilde{B}$ according to Equation (5) is sufficient to pull the resulting $H_{t}$ into the interior of this set, thereby making it invertible, except in some degenerate special cases.

In order to measure closeness, different matrix norms are possible. We choose the Frobenius norm $\|U\|_{F}=\sqrt{\sum_{i=1}^{N} \sum_{j=1}^{N} u_{i j}^{2}}$ because it is intrinsically compatible with the usual quadratic formulation of consistency results. Unfortunately, there does not appear to be any closedform solution for the minimization problems (8)-(10). We use a numerical algorithm due to Sharapov (1997, Section 3.2). For convenience, this algorithm is explained in the Appendix.

It is important to understand that this transformation makes no difference asymptotically, since the limits of $\hat{D}, \hat{A}$ and $\hat{B}$ are positive semi-definite (under Assumption 1). Therefore, the consistency of $\hat{D}, \hat{A}$ and $\hat{B}$ guarantees that of $\tilde{D}, \tilde{A}$ and $\tilde{B}$.

It should be kept in mind that the true matrices $D, A$ and $B$ (under Assumption 1) are positive semi-definite. Therefore, by forcing our estimators to be positive semi-definite, we 
are bringing them closer to the truth. The general principle is as follows: given any convex set (e.g. positive semi-definite matrices) and given any object outside this convex set, projecting this object onto the convex set brings it closer to any element of the convex set. To be fair, we are not exactly projecting $\hat{D}, \hat{A}$ and $\hat{B}$ onto the set of positive semi-definite matrices because we are preserving the diagonals. Nonetheless, this suggests that our transformation has the potential to increase parameter accuracy in every sample.

A disadvantage of our method is that it does not yield straightforward standard errors of the parameter estimates, as the transformation of the first-step matrices to positive semidefinite matrices is nonlinear and not available in closed-form. At the expense of greater computational cost, however, standard errors can be obtained by using an appropriate bootstrap method. A natural choice would be a semi-parametric bootstrap based on the fitted model. It generates bootstrap data $x_{t}^{*}, \ldots x_{T}^{*}$ in the following way:

$$
\begin{aligned}
h_{i j, t}^{*} & =\tilde{c}_{i j}+\tilde{a}_{i j} x_{i, t}^{*} x_{j, t-1}^{*}+\tilde{b}_{i j} h_{i j, t-1}^{*}, \\
x_{t}^{*} & =\left(H_{t}^{*}\right)^{1 / 2} \epsilon_{t}^{*} .
\end{aligned}
$$

Here, the $\epsilon_{t}^{*}$ are resampled from the fitted standardized residuals $\hat{\epsilon}_{t}=\hat{H}_{t}^{-1 / 2} x_{t}$, properly transformed to have sample mean equal to zero and sample covariance matrix equal to the identity. The sample covariance matrix can be used as a starting value for $H_{1}^{*}$. (To make negligible the choice of the starting value, one could actually start the generation of bootstrapped data at time $t=-M$, with $M=100$ say, and then discard the first $M+1$ values.) The following algorithm describes how to compute bootstrap standard errors for the individual parameter estimates $\tilde{c}_{i j}, \tilde{a}_{i j}$, and $\tilde{b}_{i j}$. Note that choosing $K \geq 100$ in this algorithm should be sufficient in practice; see Efron and Tibshirani (1993).

\section{Algorithm 1 (Bootstrap Standard Errors)}

1. For $k=1, \ldots, K$, generate bootstrap data $x_{1, k}^{*}, \ldots, x_{T, k}^{*}$ as described in (11)-(12).

2. Compute the estimators $\tilde{C}, \tilde{A}$, and $\tilde{B}$ on each data set to obtain bootstrap estimates $\tilde{C}_{k}^{*}, \tilde{A}_{k}^{*}$, and $\tilde{B}_{k}^{*}$, for $k=1, \ldots, K$.

3. The sample standard deviations of $\tilde{c}_{i j, k}^{*}, \tilde{a}_{i j, k}^{*}$, and $\tilde{b}_{i j, k}^{*}, k=1 \ldots K$, are the respective bootstrap standard errors of $\tilde{c}_{i j}, \tilde{a}_{i j}$, and $\tilde{b}_{i j}$.

\section{Application to International Stock Markets}

In this section, we compare the performance of several multivariate $\operatorname{GARCH}(1,1)$ covariance estimators using historical stock return data. Additionally, we compare the multivariate GARCH(1,1) estimators to other, less sophisticated estimators. (Note that a less sophisticated estimator is not necessarily an inferior estimator.) The multivariate GARCH(1,1) estimator that we developed in the previous section will be abbreviated by FlexM (for Flexible Multivariate GARCH) in the remainder of the paper. 


\subsection{Data}

We use weekly stock market data from the US, UK, France, Germany, Japan, Canada, and Switzerland, as captured by the major, broad market indices in each of these countries. The sample goes from January 1, 1975 to December 31, 2000, yielding 1,356 weekly returns observations (from the close of Wednesday to the close of next Wednesday).

We take the point of view of a US investor who does not hedge any currency risk. For each country, we thus convert weekly index prices to US dollars (using the exchange rate of the appropriate date) and then compute log-returns. To ease interpretation, the log-returns are multiplied by 100 , so they can be read as percentage returns. All data were obtained from Datastream. Summary statistics of the return data are presented in Table 1; note that the numbers for the mean and the standard deviation have been annualized.

\subsection{Competing Estimators}

For comparison, we include two popular multivariate $\operatorname{GARCH}(1,1)$ estimators and two other widely used estimators of conditional covariance matrices in the study.

\subsubsection{Constant Conditional Correlation GARCH}

Bollerslev (1990) suggested a multivariate $\operatorname{GARCH}(1,1)$ model where the conditional correlations are constant over time. To be more specific, each conditional variance $h_{i i, t}$ is modeled by a separate univariate $\operatorname{GARCH}(1,1)$ model with parameters $c_{i i}, a_{i i}$, and $b_{i i}$, respectively, and the conditional covariance between variables $x_{i}$ and $x_{j}$ at time $t$ is given by $h_{i j, t}=\rho_{i j} \sqrt{h_{i i, t} h_{j j, t}}$. Hence, there are a total of $N(N+5) / 2$ free parameters. This model gives positive definite and stationary conditional covariance matrices provided that the $\rho_{i j}$ make up a well-defined correlation matrix and the parameters $c_{i i}, a_{i i}$, and $b_{i i}$ are all nonnegative satisfying $a_{i i}+b_{i i}<1 \forall i=1, \ldots, N$. The estimation is done by maximizing the quasi-likelihood, assuming conditional normality. In the remainder of the paper, this estimator will be abbreviated by CCC.

A problem with this model is the assumption of a constant conditional correlation, which conceivably will not always hold.

\subsubsection{Diagonal BEKK GARCH}

Engle and Kroner (1995) proposed a class of multivariate GARCH models that are guaranteed to produce positive definite conditional covariance matrices. In its full generality, the corresponding GARCH(1,1) model includes all positive definite Diagonal-Vech models and suffers from its intractability problem for higher dimensions. The model most commonly used in practice is the more restrictive first-order Diagonal BEKK GARCH $(1,1)$ model given 
by:

$$
H_{t}=G^{\prime} G+E^{\prime} x_{t-1} x_{t-1}^{\prime} E+F^{\prime} H_{t-1} F,
$$

where $H_{t}$ denotes the conditional covariance matrix at time $t, x_{t}$ denotes the (column) vector of residuals at time $t, G$ is a triangular matrix, and $E$ and $F$ are diagonal matrices. Again, there are a total of $N(N+5) / 2$ free parameters and the conditional covariance matrices (that are positive semi-definite by construction) are guaranteed to be stationary if $e_{i i}^{2}+f_{i i}^{2}<1 \forall i=1, \ldots, N$. The estimation is done by maximizing the quasi-likelihood, assuming conditional normality. In the remainder of the paper, this estimator will be abbreviated by BEKK.

A problem of this model, in the notation of the general Diagonal-Vech model, are the implied constraints $a_{i j}=\sqrt{a_{i i} a_{j j}}$ and $b_{i j}=\sqrt{b_{i i} b_{j j}}$, which could easily be violated for certain data.

\subsubsection{Rolling Window}

The ever popular "rolling window" estimator simply estimates the covariance matrix at time $t$, conditional on the information available at time $t-1$, as the sample covariance matrix of the observations $\mathbf{x}_{t-k}, \ldots, \mathbf{x}_{t-1}$, where $k$ is some predetermined integer. A common choice for weekly data is $k=104$, which corresponds to a 2-year window. In the remainder of the paper, this model will be abbreviated by Window.

\subsubsection{Exponential Smoothing}

The exponential smoothing estimator is given by

$$
\hat{H}_{t}=\lambda \mathbf{x}_{t-1} \mathbf{x}_{t-1}^{\prime}+(1-\lambda) \hat{H}_{t-1},
$$

where $\lambda$ is a small, positive constant. Note that this prescription requires some suitable starting values. A common approach is to use the rolling window estimator at time $k+1$ for $\hat{H}_{1}, \ldots, \hat{H}_{k+1}$.

The exponential smoothing estimator corresponds to a multivariate Integrated Garch $(1,1)$ model with a unique autoregressive coefficient, $(1-\lambda)$, and a unique moving average coeffient, $\lambda$, for all variances and covariances. This specification is the basis of many risk measurement systems currently in use and, for example, is advocated by RiskMetrics. A commonly used value for $\lambda$ is 0.06 . In the remainder of the paper, this model will be abbreviated by RiskM.

\subsection{Estimation of the Models}

When estimating the three multivariate $\operatorname{GARCH}(1,1)$ models from the entire set of 1,356 weekly data, the estimation of the FlexM model took less than three minutes, using a proprietary optimization routine in Matlab. In contrast, the estimation of both the CCC 
and the BEKK model took over one hour, using off-the-shelf optimization routines available in Matlab. Tables 2 -5 present the estimates of the parameters of the various models. Table 3 displays bootstrap standard errors for the FlexM model.

However, we do not use these estimated models in our comparisons, as this strategy would focus on the in-sample performance of the various estimators. In-sample comparisons are not ideal for our purposes for at least two reasons. First, they are too optimistic because the entire sample is used in the fitting process before the fitted models are then applied in hindsight. Second, they tend to favor models with more degrees of freedom, so FlexM might have an unfair advantage.

We will therefore use out-of-sample comparisons in what follows. In general, the forecasts for time $t$ are made using information available up to time $t-1$ only. The parameter estimates of the multivariate $\mathrm{GARCH}(1,1)$ models are updated every four weeks to reduce the computational burden for BEKK and CCC. All forecasts start at time $t=601$.

\subsection{Forecast Criteria}

The real test for a multivariate $\operatorname{GARCH}(1,1)$ model is to compare its estimated, or forecasted conditional covariance matrix to the true, realized matrix. The latter is unobservable but a proxy can be constructed. A common and successful approach, termed integrated volatility, is to use cumulative cross-products of intraday return residuals over the forecast horizon; for example, see Andersen, Bollerslev, and Lange (1999), henceforth ABL, or Andersen, Bollerslev, Diebold, and Labys (2001). Unfortunately, we only have daily return data available but the same methodology can be applied to them; this results in a less precise but still useful proxy. We consider forecast horizons of 1, 2, and 4 weeks. Note that there are standard formulas to compute the the 2-week and 4-week forecasts for multivariate GARCH models, given the 1-week forecast and the estimated model at time $t-1$; for example, see ABL. To compute the 2-week and 4-week forecasts for RiskM and Window, we

simply multiply the 1 -week forecasts by the forecast horizon. Denote by $\hat{H}_{t, k}$ the estimated conditional covariance matrix, based on the information available at time $t-1$, for the $k$ week forecast horizon; in this notation $\hat{H}_{t, 1}$ corresponds to $\hat{H}_{t}$, the 1-week forecast. Also, let $\Sigma_{t, k}$ be the cumulative cross-products of daily return residuals during that period. The typical elements of these two matrices are denoted by $\hat{h}_{i j, t, k}$ and $\sigma_{i j, t, k}$, respectively. As do $\mathrm{ABL}$, we consider the following two criteria to judge the quality of the volatility forecasts:

$$
\begin{aligned}
\operatorname{RMSE}_{k} & =\left[\frac{1}{N^{2}} \sum_{i, j} E\left(\hat{h}_{i j, t, k}-\sigma_{i j, t, k}\right)^{2}\right]^{1 / 2} \\
\mathrm{MAD}_{k} & =\frac{1}{N^{2}} \sum_{i, j} E\left|\hat{h}_{i j, t, k}-\sigma_{i j, t, k}\right|
\end{aligned}
$$

$\mathrm{RMSE}_{k}$ and $\mathrm{MAD}_{k}$ are multivariate versions of root mean squared error and mean absolute deviation, respectively. Criteria based on absolute deviations are sometimes preferred, since they are more robust and less affected by a few large outliers; e.g., ABL. 
Table 6 reports estimates of the two criteria at the different forecast horizons. There are 6 comparisons altogether ( 2 criteria and 3 horizons). FlexM is best 4 times - for both criteria at the 1-week and 2-week horizons - and CCC is best 2 times - for both criteria at the 4-week horizon. RiskM and Window are always worse than the multivariate GARCH models.

\subsection{Standardized Residuals}

Consider the standardized residuals $\epsilon_{t}=H_{t}^{-1 / 2} x_{t}$, where $H_{t}$ is the true conditional covariance matrix at time $t$. Obviously, the $\epsilon_{t}$ have constant conditional covariance matrix equal to the identity and the cross-products $\epsilon_{t} \epsilon_{t}^{\prime}$ are uncorrelated over time. It is therefore natural to test for any 'leftover' autocorrelation in the cross-products $\hat{\epsilon}_{t} \hat{\epsilon}_{t}^{\prime}$, where $\hat{\epsilon}_{t}=\hat{H}_{t}^{-1 / 2} x_{t}$ and $\hat{H}_{t}$ is the estimated conditional covariance matrix at time $t$.

A standard test for serial correlation in a univariate time series $\left\{y_{t}\right\}$ is the Ljung-Box test. The test statistic is:

$$
L B(k)=T \sum_{l=1}^{k} \frac{T+2}{T-l} \hat{\rho}(l),
$$

where $\hat{\rho}(l)$ is the sample autocorrelation of order $l$ and $k$ is an integer which is small compared to the sample size $T$. The commonly used asymptotic null distribution is $\chi_{k}^{2}$, the Chi-square distribution with $k$ degrees of freedom.

There are, however, two problems with applying this test for our purposes. A general problem is that the asymptotic null distribution is only correct under the additional assumption of i.i.d. data. If the series $\left\{y_{t}\right\}$ is uncorrelated but dependent, the $\chi_{k}^{2}$ approximation can be arbitrarily misleading (Romano and Thombs, 1996). Another problem is that the test is designed for univariate series and not series of $N \times N$ matrices. We address these two problems simultaneously by suggesting a "combined" test statistic that takes into account all cross-product elements at once and by constructing a test that, under the null, only requires that the cross-products are uncorrelated rather than i.i.d..

The combined test statistic we suggest is:

$$
L B_{\text {comb. }}(k)=\sum_{i \leq j} L B_{i j}(k)
$$

where $L B_{i j}(k)$ is the univariate Ljung-Box test statistic computed from the series $\left\{\hat{\epsilon}_{i, t} \hat{\epsilon}_{j, t}\right\}$. To assess the evidence against the null hypothesis, we compute the $P$-value based on the subsampling method. To this end, let $L B_{\text {comb.t,b }}(k)$ be the combined test statistic based on the stretch of data $\left\{\hat{\epsilon}_{t}, \ldots, \hat{\epsilon}_{t+b-1}\right\}$, for $t=1, \ldots, T-b+1$. Here, the block size $b$ is an integer smaller than $T$. The subsampling $P$-value is then given as:

$$
P V_{\text {Sub }}=\frac{\#\left\{L B_{\text {comb. }, t, b}(k) \geq L B_{\text {comb. }}(k)\right\}}{T-b+1} .
$$

By arguments analogous to the ones of Romano and Thombs (1996), it can easily be shown 
that this test is consistent if the cross-products are uncorrelated but dependent. For more details about the general use of subsampling tests with dependent data, the reader is referred to Politis, Romano, and Wolf (1999, Chapter 3). The block size $b$ needs to satisfy the asymptotic conditions $b \rightarrow \infty$ and $b / T \rightarrow 0$; some methods for chosing $b$ in practice are given in Politis, Romano, and Wolf (1999, Chapter 9).

Table 7 presents the test statistic and corresponding $P$-value for the five models, using $k=12$ and $b=100$; the results are similar for other values of $k$ and $b$. FlexM has the smallest test statistic and is the only model that is not rejected at any conventional level; its $P$-value is 0.72 , the one for RiskM is 0.01 , and all the others are 0.

\subsection{Value-at-Risk}

An important use of the conditional covariance matrix is in calculations of Value-at-Risk (VaR). A large number of methods to compute VaR have been suggested and are currently employed, such as historical simulation, RiskMetrics, Monte Carlo, GARCH, nonparametric quantile regressions, and methods based on extreme value theory. We certainly do not aim to settle the dispute as to which method is "best," and it stands to reason that a uniformly best method does not exist. However, GARCH methods are very popular among practitioners and tend to perform well. (In particular, recent claims that they are dominated by methods based on extreme value theory do not seem to be substantiated; for example, see Lee and Saltoğlu; 2001.)

If a single portfolio is considered, it makes more sense to fit a univariate $\operatorname{GARCH}(1,1)$ model to the corresponding return series and base any $\mathrm{VaR}$ calculations on this model. On the other hand, if a number of different portfolios based on the same universe of $N$ assets are considered (as is the case with different traders of an investment bank, say), it is common practice to base the individual VaR calculations on a single estimate of the conditional covariance matrix of all $N$ assets. This also allows computing marginal contributions to risk of each position and evaluating the impact of hedges. Hence, multivariate GARCH is certainly relevant to risk management applications.

In our tests, we consider the following four portfolios based on the seven market indices that make up our data:

- U.S. Portfolio: U.S. only.

- North-American Portfolio: U.S. and Canada equally weighted.

- European Portfolio: U.K., France, Germany, and Switzerland equally weighted.

- World Portfolio: all seven countries equally weighted.

We use the estimated conditional covariance matrix to compute the one-week-ahead VaR at levels $1 \%$ and $5 \%$. In order to try to fit the tails of the return distributions and to match 
the theoretical VaR levels, we assume a conditional $t$-distribution. To be more specific, let the portfolio be represented by the vector of weights, $w$. The estimated conditional variance of the portfolio at time $t$ is then given by:

$$
\hat{h}_{w, t}=w^{\prime} \hat{H}_{t} w
$$

At time $t-1$, we condition on the past portfolio returns and their corresponding estimated conditional variances to choose the degrees of freedom, $\nu^{*}$, that maximize the likelihood:

$$
\prod_{s=1}^{t-1} \frac{\Gamma\left(\frac{\nu+1}{2}\right)}{\sqrt{\pi(\nu-2) \hat{h}_{w, s}} \Gamma(\nu / 2)}\left[1+\frac{\left(w^{\prime} x_{s}\right)^{2}}{(\nu-2) \hat{h}_{w, s}}\right]^{-(\nu+1) / 2}
$$

over $\nu$, where $\Gamma(\cdot)$ denotes the "gamma" function. Note that the standard formula for the $t$-distribution has been modified by the scale factor $\hat{h}_{w, s}(\nu-2) / \nu$, where the degree-offreedom adjustment is designed so that $\hat{h}_{w, s}$ is exactly equal to the conditional variance of $w^{\prime} x_{s}$. Having thus found $\nu^{*}$, the $1 \% \mathrm{VaR}$ at time $t$ is finally computed as

$$
t_{\nu^{*}, 0.01} \sqrt{\hat{h}_{w, t}\left(\nu^{*}-2\right) / \nu^{*}} \text {. }
$$

Here, $t_{\nu^{*}, 0.01}$ denotes the 0.01 quantile of the $t$-distribution with $\nu^{*}$ degrees of freedom. An analogous computation yields the $5 \%$ VaR.

For a certain portfolio and for a given level, define the hit variable:

$$
h_{t}=I\left\{w^{\prime} x_{t}<\widehat{\mathrm{VaR}}_{t}\right\}
$$

where $I\{\cdot\}$ is the indicator function and $\widehat{\mathrm{VaR}}_{t}$ is the estimated $\mathrm{VaR}$ at time $t$. If the model to calculate the VaR is correctly specified, the series $\left\{h i t_{t}\right\}$ should be uncorrelated over time and have expected value equal to the desired confidence level.

Table 8 presents the sample means (or "hit rates") and the Ljung-Box $P$-values for autocorrelation of the hit series for the various methods, portfolios, and VaR levels. The $P$-values are based on the first $k=12$ sample autocorrelations. Since the hit series are univariate and given the fact that a (stationary) $\{0,1\}$ series is uncorrelated if and only if it is independent, it is safe to use the asymptotic Chi-square approximation to compute the $P$-values here.

The hit rates are all reasonably close to the target levels, although they tend to be a bit larger on average. There is no clear winner, nor a clear loser in terms of the hit rates. Judging the serial correlation of the hit series $\left\{h i t_{t}\right\}$, it is seen that RiskM performs best, since all its $P$-values are above 0.1 . We conclude that FlexM is somewhat better than the other GARCH models. 


\subsection{Portfolio Selection}

Another important application of the conditional covariance matrix is as an input to the Markowitz (1952) portfolio selection method. Hence, we examine the gains from international diversification obtained by taking into account the time changing nature of the covariance matrix. In order to avoid having to specify the vector of conditional expected returns, which is more a task of the portfolio manager than a statistical problem, we focus on constructing the (global) minimum variance portfolio, allowing for short sales.

Table 9 shows the realized (annualized) standard deviation of the returns of the conditional minimum variance portfolio over the entire sample period obtained from the three GARCH models, the RiskMetrics method, and the Rolling Window method. It compares them to the standard deviation of the US stock market, of the equal-weighted portfolio of the seven stock markets, and of the unconditional minimum variance portfolio obtained from the sample covariance matrix at $t=1,356$. (The last portfolio would be infeasible but we include it nevertheless.) Not surprisingly, fully investing in the US stock market yields the highest standard deviation, followed by the equal-weighted world portfolio and the unconditional minimum variance portfolio. All three GARCH model provide a significant improvement, with FlexM being the best. Window is comparable to the unconditional minimum variance portfolio and RiskM is worse than even the equal-weighted portfolio.

\section{Conclusion}

In this paper, we have developed an estimation procedure for the general Diagonal-Vech formulation of the Multivariate $\operatorname{GARCH}(1,1)$ model. Our procedure is the first to be computationally feasible for dimensions $N>5$, without constraining the coefficient matrices. Our method proceeds in two steps: first, we decentralize the problem by estimating separately $N$ univariate and $N(N-1) / 2$ bivariate GARCH models, all of which are computationally feasible problems; second, we bring together these results to form $N$-dimensional matrices of parameter estimates, which we transform in order to ensure the positive semi-definiteness of the conditional covariance matrices. In doing so, we avoid having to impose additional restrictions, which has been the common approach so far in the multivariate GARCH literature. In addition, our method is computationally far less demanding than traditional multivariate models, which is an important advantage if the sample size is large, as would be the case with high-frequency data.

We apply our procedure to 25 years of weekly data on 7 major international stock markets and compare it to two popular traditional multivariate $\operatorname{GARCH}(1,1)$ models, namely the constant conditional correlation model and the Diagonal BEKK model and two widelyused, albeit less sophisticated, estimators, namely the Rolling Window estimator and the exponential smoothing estimator. Using a number of criteria, such as forecast accuracy, persistence of standardized residuals, precision of Value-at-Risk estimates, and optimal portfolio selection, we find that the Flexible Multivariate GARCH method does indeed offer improved performance. The use of high-frequency data, which undoubtedly will increase in 
the future, should make our procedure even more attractive.

Direct applications of this method involve portfolio selection and tests of asset pricing models such as the International CAPM, and risk measurement uses such as Value-atRisk. An interesting topic left for future research is an extension to asymmetric multivariate $\operatorname{GARCH}(1,1)$. 


\section{Appendix: Minimization of the Frobenius norm}

\section{A.1 Problem Formulation}

Given a symmetric matrix $A$ with the property $\operatorname{diag}(A)>0$, find a symmetric, positive semi-definite matrix $M$ with $\operatorname{diag}(M)=\operatorname{diag}(A)$ that minimizes $\|A-M\|_{F}$, where $\|\cdot\|_{F}$ is the Frobenius norm.

\section{A.2 Numerical Solution}

Write the matrix $A$ and the current approximation $M$ to the solution of the above problem as

$$
A=\left(\begin{array}{cc}
a_{11} & a^{T} \\
a & \bar{A}
\end{array}\right) \quad M=\left(\begin{array}{cc}
a_{11} & m^{T} \\
m & \bar{M}
\end{array}\right)
$$

and let the conditions of the problem be satisfied (that is, $\operatorname{diag}(M)=\operatorname{diag}(A)$ and $\left.M=M^{T} \geq 0\right)$. For a matrix of the form

$$
P=\left(\begin{array}{cc}
\rho & x^{T} \\
0 & I_{n-1}
\end{array}\right)
$$

(where $I_{n-1}$ is identity), we can introduce the next iterate by

$$
\check{M}=P M P^{T}=\left(\begin{array}{cc}
\rho^{2} a_{11}+2 \rho x^{T} m+x^{T} M x & \rho m^{T}+x^{T} \bar{M} \\
\rho m+\bar{M} x & \bar{M}
\end{array}\right) .
$$

If we enforce the condition

$$
\rho^{2} a_{11}+2 \rho x^{T} m+x^{T} M x=a_{11}
$$

then the new approximation $\check{M}$ satisfies the conditions of the problem: $\check{M}=\check{M}^{T} \geq 0$ and $\operatorname{diag}(\check{M})=\operatorname{diag}(A)$.

If (18) is satisfied we have

$$
\|A-\check{M}\|_{F}-\|A-M\|_{F}=2\|a-(\rho m+\bar{M} x)\|_{2}^{2}-2\|a-m\|_{2}^{2},
$$

therefore choosing $x$ and $\rho$ that minimize $\|a-(\rho m+\bar{M} x)\|_{2}^{2}$ from (17) we get $\check{M}$ that minimizes $\|A-\check{M}\|_{F}$, satisfies the conditions of the problem, and is obtained form the previous approximation $M$ by changing its first row and column. The extension to the $i$ th column and row is obvious.

Remark 1 The convexity of the problem implies that the solution matrix $M$ is singular, that is, lies on the boundary of the feasible region. Since $\operatorname{det}(\check{M})=\rho^{2} \operatorname{det}(M)$, we can make the iterates stay within the interior of the feasible region by initializing the process with 
a nonsingular matrix and choosing $\rho$ to be bounded away from zero. Later on we treat $\rho$ as a chosen constant between zero and one, so the iterates become singular not faster then exponentially. In numerical examples, $\rho$ is chosen to be 0.5 .

One step of the iterative procedure becomes

$$
\min _{x}\|a-(\rho m+\bar{M} x)\|_{2}^{2}
$$

subject to (18), or introducing

$$
b=a-\rho m
$$

it becomes

$$
\min _{x}\|\bar{M} x-b\|_{2}^{2}
$$

still subject to (18).

The Lagrangian of this subproblem is

$$
L(x, \lambda)=\|\bar{M} x-b\|_{2}^{2}+\lambda\left(\rho^{2} a_{11}+2 \rho x^{T} m+x^{T} M x-a_{11}\right)
$$

and the optimality conditions are

$$
F(x)=\rho^{2} a_{11}+2 \rho x^{T} m+x^{T} M x-a_{11}=0
$$

and

$$
\nabla_{x} L(x, \lambda)=\overline{0}
$$

which can be written as

$$
\bar{M}^{2} x-\bar{M} b+\lambda \rho m+\lambda \bar{M} x=0 .
$$

For any $\lambda$, Equation (20) can be solved for $x$ :

$$
x(\lambda)=\left(\bar{M}^{2}+\lambda \bar{M}\right)^{-1}(\bar{M} b-\lambda \rho m)
$$

and

$$
F(\lambda)=F(x(\lambda))=0
$$

can be solved by the Newton's method:

$$
\lambda \Leftarrow \lambda-\frac{F(\lambda)}{F_{\lambda}(\lambda)}
$$

The analytic expression for $F_{\lambda}(\lambda)$ can be obtained from

$$
F_{\lambda}(\lambda)=\nabla_{x} F(x) \cdot x_{\lambda}=2(\rho m+\bar{M} x)^{T} x_{\lambda}
$$

By differentiating (20) in $\lambda$ we get

$$
\bar{M}^{2} x_{\lambda}+\rho m+\bar{M} x+x \bar{M} x_{\lambda}=0,
$$


therefore

$$
x_{\lambda}=-\left(\bar{M}^{2}+\lambda \bar{M}\right)^{-1}(\rho m+\bar{M} x) .
$$

inserting in (23) we get

$$
F_{\lambda}(\lambda)=-2(\rho m+\bar{M} x)^{T}\left(\bar{M}^{2}+\lambda \bar{M}\right)^{-1}(\rho m+\bar{M} x) .
$$

We can summarize the solution of the subproblem as:

- Initialize $\lambda($ say $\lambda=0)$

- Compute $x$ by (21)

- Compute $F(\lambda)$ and $F_{\lambda}(\lambda)$ using (19) and (24)

- Update $\lambda$ using Newton's step (22)

- Recur the Newton's procedure

Remark 2 Steps (19) and (24) involve the inverse of $\bar{M}^{2}+\lambda \bar{M}$ which is singular if $\bar{M}$ is. Restricting $\rho$ to be a nonzero constant results in nonsingular $\bar{M}$ unless it is a solution; see Remark 1.

A MATLAB routine implementing this procedure has been written Ilya Sharapov and is available from the authors upon request.

\section{A.3 Numerical Tests}

Extensive simulation tests of the numeric routing have been implemented. Numerical convergence is typically obtained after one or two iterations with minimal error. The results to these tests are not presented for brevity but can be obtained from the authors upon request. 


\section{References}

Andersen, T.G., T. Bollerslev, F.X. Diebold, and P. Labys (2001). The Distribution of Realized Exchange Rate Volatility. Journal of the American Statistical Association 96, $42-55$.

Andersen, T.G., T. Bollerslev and S. Lange (1999). Forecasting Financial Market Volatility: Sample Frequency Vis-à-vis Forecast Horizon. Journal of Empirical Finance 6, 457-477.

Bollerslev, T. (1990). Modelling the Coherence in Short-run Nominal Exchange Rates: A Multivariate Generalized ARCH Model. Review of Economics and Statistics 72, 498-505.

Bollerslev, T., R.F. Engle and J. M. Wooldridge (1988). A Capital Asset Pricing Model With Time-Varying Covariances. Journal of Political Economy 96, 116-131.

Campbell, J.Y., A.W. Lo and A.C. MacKinlay. (1997). The Econometrics of Financial Markets. Princeton University Press.

Ding, Z. and R.F. Engle (1994). Large Scale Conditional Covariance Matrix Modeling, Estimation and Testing. Working Paper, Department of Economics, UCSD.

Efron, B. and R.J. Tibshirani (1993). An Introduction to the Bootstrap. Chapman \& Hall, New York.

Engle, R.F. (2000). Dynamic Conditional Correlation - A Simple Class of Multivariate GARCH Models. Working Paper, Department of Economics, UCSD.

Engle, R.F. and K. Kroner (1995). Multivariate Simultaneous GARCH. Econometric Theory 11, 122-150.

Engle, R.F. and J. Mezrich (1996). GARCH for Groups. Risk 9, 36-40.

Lee, T.-H. and B. Saltoğlu (2001). Evaluating Predictive Performance of Value-at-Risk Models in Emerging Markets: A Reality Check. Working Paper, Department of Economics, University of California Riverside.

Markowitz, H. (1952). Portfolio Selection. Journal of Finance 7, 77-91.

Politis, D.N., J.P Romano, and M. Wolf (1999). Subsampling. Springer, New York.

Romano, J.P. and L.A. Thombs (1996). Inference for Autocorrelations under Weak Assumptions. Journal of the American Statistical Association 91, 590-600.

Sharapov, I. (1997). Advances in Multigrid Optimization Methods with Applications. 
Ph.D. Thesis, CAM Report 97-31, Department of Mathematics, UCLA.

Styan, G.P.H. (1973). Hadamard Products and Multivariate Statistical Analysis. Linear Algebra and Its Applications 6, 217-240. 
Table 1: Summary Statistics of Log-Returns. This table presents the summary statistics for the weekly percentage log-returns of seven different stock markets. The local currency returns were transformed into US Dollar returns by the appropriate exchange rate, they correspond to the returns obtained by a US investor who does not hedge currency risk. The sample includes 1,356 observations from January 1, 1975 to December 31, 2000, obtained from Datastream. The numbers for the mean and the standard deviation are annualized.

\begin{tabular}{lccccccc}
\hline & USA & UK & France & Germany & Japan & Canada & Switzerland \\
& & & & & & & \\
Mean & 15.16 & 17.85 & 15.91 & 12.14 & 11.69 & 11.27 & 15.23 \\
SD & 15.10 & 19.71 & 20.57 & 17.66 & 20.44 & 15.62 & 16.48 \\
Skewness & -0.60 & 0.25 & -0.49 & -0.31 & 0.11 & -0.47 & -0.60 \\
Kurtosis & 6.82 & 9.01 & 5.29 & 4.72 & 4.80 & 6.54 & 6.95 \\
& & & & & & & \\
\hline
\end{tabular}


Table 2: Parameter Estimates of the FlexM Model. This table presents the estimated parameters of the Flexible Multivariate (FlexM) GARCH $(1,1)$ model based on the entire sample. The model is developed and described in Section 2. As the matrices are symmetric, only the lower triangular parts are displayed to enhance readability.

\begin{tabular}{|c|c|c|c|c|c|c|}
\hline USA & UK & France & Germany & Japan & Canada & Switzerland \\
\hline \multicolumn{7}{|c|}{$\tilde{C}$} \\
\hline \multicolumn{7}{|l|}{0.1448} \\
\hline 0.1292 & 0.1641 & & & & & \\
\hline 0.1560 & 0.2120 & 0.4410 & & & & \\
\hline 0.0744 & 0.1270 & 0.1752 & 0.1783 & & & \\
\hline 0.0627 & 0.1119 & 0.1666 & 0.1160 & 0.1858 & & \\
\hline 0.1813 & 0.1840 & 0.1876 & 0.0950 & 0.0732 & 0.2929 & \\
\hline 0.1495 & 0.2062 & 0.2798 & 0.2575 & 0.1958 & 0.1817 & 0.5271 \\
\hline \multicolumn{7}{|c|}{$\tilde{A}$} \\
\hline \multicolumn{7}{|l|}{0.0861} \\
\hline 0.0499 & 0.0710 & & & & & \\
\hline 0.0533 & 0.0700 & 0.1037 & & & & \\
\hline 0.0641 & 0.0680 & 0.0940 & 0.0918 & & & \\
\hline 0.0475 & 0.0405 & 0.0565 & 0.0600 & 0.0772 & & \\
\hline 0.0839 & 0.0474 & 0.0600 & 0.0768 & 0.0565 & 0.1162 & \\
\hline 0.0517 & 0.0629 & 0.0876 & 0.0820 & 0.0521 & 0.0567 & 0.0783 \\
\hline \multicolumn{7}{|c|}{$\tilde{B}$} \\
\hline \multicolumn{7}{|l|}{0.8835} \\
\hline 0.8886 & 0.9066 & & & & & \\
\hline 0.8606 & 0.8714 & 0.8426 & & & & \\
\hline 0.8802 & 0.8880 & 0.8595 & 0.8808 & & & \\
\hline 0.8896 & 0.9018 & 0.8700 & 0.8873 & 0.9012 & & \\
\hline 0.8509 & 0.8616 & 0.8319 & 0.8497 & 0.8601 & 0.8233 & \\
\hline 0.8482 & 0.8588 & 0.8292 & 0.8469 & 0.8573 & 0.8198 & 0.8179 \\
\hline
\end{tabular}


Table 3: Standard Errors of the FlexM Model. This table presents bootstrap standard errors corresponding to the parameter estimates of Table 2. The standard errors were computed as outlined in Algorithm 1, using $K=100$. As the matrices are symmetric, only the lower triangular parts are displayed to enhance readability.

\begin{tabular}{|c|c|c|c|c|c|c|}
\hline USA & UK & France & Germany & Japan & Canada & Switzerland \\
\hline \multicolumn{7}{|c|}{$\tilde{C}$} \\
\hline \multicolumn{7}{|l|}{0.0862} \\
\hline 0.0497 & 0.1286 & & & & & \\
\hline 0.0554 & 0.0903 & 0.2221 & & & & \\
\hline 0.0354 & 0.0550 & 0.0675 & 0.0869 & & & \\
\hline 0.0360 & 0.0575 & 0.0610 & 0.0456 & 0.0948 & & \\
\hline 0.0759 & 0.0755 & 0.0860 & 0.0516 & 0.0439 & 0.1406 & \\
\hline 0.0957 & 0.1505 & 0.1898 & 0.1508 & 0.1512 & 0.1068 & 0.5059 \\
\hline \multicolumn{7}{|c|}{$\tilde{A}$} \\
\hline \multicolumn{7}{|l|}{0.0271} \\
\hline 0.0183 & 0.0220 & & & & & \\
\hline 0.0186 & 0.0164 & 0.0294 & & & & \\
\hline 0.0169 & 0.0171 & 0.0206 & 0.0264 & & & \\
\hline 0.0177 & 0.0146 & 0.0149 & 0.0138 & 0.0191 & & \\
\hline 0.0245 & 0.0179 & 0.0197 & 0.0183 & 0.0172 & 0.0369 & \\
\hline \multirow[t]{2}{*}{0.0212} & 0.0228 & 0.0255 & 0.0284 & 0.0177 & 0.0244 & 0.0458 \\
\hline & & & $\tilde{B}$ & & & \\
\hline \multicolumn{7}{|l|}{0.0379} \\
\hline 0.0319 & 0.0337 & & & & & \\
\hline 0.0419 & 0.0339 & 0.0461 & & & & \\
\hline 0.0362 & 0.0288 & 0.0313 & 0.0332 & & & \\
\hline 0.0390 & 0.0319 & 0.0350 & 0.0283 & 0.0254 & & \\
\hline 0.0428 & 0.0420 & 0.0537 & 0.0460 & 0.0524 & 0.0551 & \\
\hline 0.0890 & 0.0873 & 0.0848 & 0.0853 & 0.0890 & 0.0913 & 0.1320 \\
\hline
\end{tabular}


Table 4: Parameter Estimates of the CCC Model. This table presents the estimated parameters of the Constant Conditional Correlation (CCC) $\operatorname{GARCH}(1,1)$ model based on the entire sample. The model is described in Subsubsection 3.2.1.

\begin{tabular}{|c|c|c|c|c|c|c|}
\hline USA & UK & France & Germany & Japan & Canada & Switzerland \\
\hline \multicolumn{7}{|c|}{$c_{i i}$} \\
\hline 0.1850 & 0.0733 & 0.3495 & 0.1514 & 0.2356 & 0.2992 & 0.5743 \\
\hline \multicolumn{7}{|c|}{$a_{i i}$} \\
\hline 0.0700 & 0.0520 & 0.0731 & 0.0446 & 0.0727 & 0.0898 & 0.0467 \\
\hline \multicolumn{7}{|c|}{$b_{i i}$} \\
\hline 0.8872 & 0.9379 & 0.8819 & 0.9278 & 0.8977 & 0.8443 & 0.8367 \\
\hline \multicolumn{7}{|c|}{ Correlation Matrix } \\
\hline \multicolumn{7}{|l|}{1.0000} \\
\hline 0.4392 & 1.0000 & & & & & \\
\hline 0.3572 & 0.4888 & 1.0000 & & & & \\
\hline 0.3302 & 0.4623 & 0.5643 & 1.0000 & & & \\
\hline 0.2469 & 0.3369 & 0.3373 & 0.3688 & 1.0000 & & \\
\hline 0.6753 & 0.4352 & 0.3459 & 0.3267 & 0.2244 & 1.0000 & \\
\hline 0.3655 & 0.4852 & 0.5299 & 0.7009 & 0.3891 & 0.3450 & 1.0000 \\
\hline
\end{tabular}


Table 5: Parameter Estimates of the BEKK Model. This table presents the estimated parameters of the Diagonal BEKK (BEKK) GARCH $(1,1)$ model based on the entire sample. The model is described in Subsubsection 3.2.2. Note that $G$ is a lower triangular matrix, so the elements not displayed are equal to zero.

\begin{tabular}{|c|c|c|c|c|c|c|}
\hline USA & UK & France & Germany & Japan & Canada & Switzerland \\
\hline \multicolumn{7}{|c|}{$G$} \\
\hline \multicolumn{7}{|l|}{0.3034} \\
\hline 0.1004 & 0.1662 & & & & & \\
\hline 0.1508 & 0.2863 & 0.2634 & & & & \\
\hline 0.1291 & 0.2558 & 0.0215 & 0.2696 & & & \\
\hline 0.0842 & 0.1154 & 0.0374 & 0.0561 & 0.3300 & & \\
\hline 0.2408 & 0.0831 & -0.0329 & -0.0270 & -0.0060 & 0.2485 & \\
\hline 0.2039 & 0.3923 & -0.0519 & 0.0905 & 0.0557 & -0.0620 & 0.2302 \\
\hline \multicolumn{7}{|c|}{$\operatorname{Diag}(E)$} \\
\hline 0.1446 & 0.1283 & 0.2141 & 0.2050 & 0.1709 & 0.1567 & 0.1872 \\
\hline \multicolumn{7}{|c|}{$\operatorname{Diag}(F)$} \\
\hline 0.9786 & 0.9885 & 0.9657 & 0.9655 & 0.9769 & 0.9729 & 0.9557 \\
\hline
\end{tabular}


Table 6: Forecast Criteria for Covariance Matrices. This tables compares the forecasted conditional covariance matrices with the realized integrated volatility covariance matrices computed from daily data that serve as a proxy for the true but unobservable conditional covariance matrices. The criteria RMSE and MAD are defined in (13)-(14). All forecasts are out-of-sample. Forecasts start at week $t=601$.

\begin{tabular}{|c|c|c|}
\hline Model & RMSE & MAD \\
\hline \multicolumn{3}{|c|}{ 1-Week Horizon } \\
\hline FlexM & 9.73 & 2.96 \\
\hline $\mathrm{CCC}$ & 9.88 & 3.01 \\
\hline BEKK & 9.90 & 3.09 \\
\hline RiskM & 9.98 & 3.31 \\
\hline Window & 10.02 & 3.42 \\
\hline \multicolumn{3}{|c|}{ 2-Week Horizon } \\
\hline FlexM & 15.48 & 5.13 \\
\hline CCC & 15.70 & 5.22 \\
\hline BEKK & 15.74 & 5.37 \\
\hline RiskM & 16.07 & 5.88 \\
\hline Window & 16.03 & 6.09 \\
\hline \multicolumn{3}{|c|}{ 4-Week Horizon } \\
\hline FlexM & 17.42 & 8.90 \\
\hline $\mathrm{CCC}$ & 17.09 & 8.71 \\
\hline BEKK & 17.48 & 9.37 \\
\hline RiskM & 26.14 & 10.82 \\
\hline Window & 25.61 & 11.10 \\
\hline
\end{tabular}


Table 7: Standardized Residuals. This tables presents test results for left-over autocorrelation in the fitted standardized residuals. The test statistic is the combined LjungBox statistic defined in (15) using the first $k=12$ sample autocorrelations. The $P$-value for the null hypothesis of no autocorrelation is obtained by applying the subsampling method with block size $b=100$, as detailed in the discussion following Equation (15). All fitted standardized residuals are out-of-sample and are computed starting at week $t=601$.

\begin{tabular}{lcc}
\hline \multicolumn{1}{r}{ Model } & Test Statistic & $P$-value \\
FlexM & 275.4 & 0.72 \\
CCC & 640.1 & 0.00 \\
BEKK & 760.1 & 0.00 \\
RiskM & 386.7 & 0.01 \\
Window & 845.3 & 0.00 \\
& & \\
\hline
\end{tabular}


Table 8: Value-at-Risk. This table compares VaR calculations at levels $1 \%$ and 5\%, assuming a conditional $t$-distribution (suitably normalized). The hit rates are the sample means of the hit series defined in (16) and should be close to the nominal level. The $P$-value corresponds to the null hypothesis of no autocorrelation in the hit series and is obtained from the usual Chi-square approximation of the univariate Ljung-Box test statistic using the first $k=12$ sample autocorrelations. All VaR calculations are out-of-sample and start at week $t=601$.

\begin{tabular}{|c|c|c|c|c|}
\hline Model & Hit Rate for $1 \%$ & Hit Rate for $5 \%$ & $P$-value for $1 \%$ & $P$-value for $5 \%$ \\
\hline \multicolumn{5}{|c|}{ U.S. Portfolio } \\
\hline FlexM & 0.027 & 0.068 & 0.71 & 0.82 \\
\hline $\mathrm{CCC}$ & 0.027 & 0.068 & 0.64 & 0.93 \\
\hline BEKK & 0.018 & 0.068 & 0.36 & 0.99 \\
\hline RiskM & 0.030 & 0.066 & 0.85 & 0.83 \\
\hline Window & 0.029 & 0.068 & 0.78 & 0.24 \\
\hline \multicolumn{5}{|c|}{ North-American Portfolio } \\
\hline FlexM & 0.015 & 0.070 & 0.90 & 0.86 \\
\hline $\mathrm{CCC}$ & 0.015 & 0.070 & 0.00 & 0.68 \\
\hline BEKK & 0.015 & 0.068 & 0.07 & 0.79 \\
\hline RiskM & 0.027 & 0.066 & 0.61 & 0.44 \\
\hline Window & 0.028 & 0.062 & 0.01 & 0.51 \\
\hline \multicolumn{5}{|c|}{ European Portfolio } \\
\hline FlexM & 0.010 & 0.054 & 0.01 & 0.18 \\
\hline $\mathrm{CCC}$ & 0.016 & 0.060 & 0.08 & 0.01 \\
\hline BEKK & 0.011 & 0.054 & 0.04 & 0.09 \\
\hline RiskM & 0.015 & 0.058 & 0.55 & 0.30 \\
\hline Window & 0.017 & 0.052 & 0.00 & 0.00 \\
\hline \multicolumn{5}{|c|}{ World Portfolio } \\
\hline FlexM & 0.016 & 0.063 & 0.03 & 0.44 \\
\hline $\mathrm{CCC}$ & 0.015 & 0.069 & 0.00 & 0.00 \\
\hline BEKK & 0.016 & 0.053 & 0.03 & 0.00 \\
\hline RiskM & 0.020 & 0.065 & 0.18 & 0.26 \\
\hline Window & 0.021 & 0.061 & 0.02 & 0.00 \\
\hline
\end{tabular}


Table 9: Standard Deviation of Portfolio Returns. This table presents (annualized) standard deviations of various portfolios. The Minimum Variance Portfolios aim to construct the portfolio with globally minimum variance; no restriction on the expected return is made and short sales are allowed. All portfolios are constructed starting at week $t=601$.

\begin{tabular}{lc}
\hline US Portfolio & 15.87 \\
Equal-Weighted World Portfolio & 13.33 \\
Unconditional Minimum Variance Portfolio & 12.91 \\
FlexM Minimum Variance Portfolio & 12.32 \\
CCC Minimum Variance Portfolio & 12.53 \\
BEKK Minimum Variance Portfolio & 12.54 \\
RiskM Minimum Variance Portfolio & 13.37 \\
Window Minimum Variance Portfolio & 12.89 \\
\hline
\end{tabular}

